\title{
Early risk prediction in idiopathic versus connective tissue disease-associated pulmonary arterial hypertension: call for a refined assessment
}

\author{
Clara Hjalmarsson (10 ${ }^{1}$, Barbro Kjellström $\mathbb{1}^{2,3}$, Kjell Jansson ${ }^{4}$, Magnus Nisell ${ }^{5,6}$, David Kylhammar ${ }^{4}$, \\ Mohammad Kavianipour ${ }^{7}$, Göran Rådegran $\mathbb{1}^{8}$, Stefan Söderberg $\mathbb{1}^{9}$, Gerhard Wikström ${ }^{10}$, \\ Dirk M. Wuttge ${ }^{11}$ and Roger Hesselstrand ${ }^{11}$
}

\begin{abstract}
${ }^{1}$ Dept of Cardiology, Sahlgrenska Academy, University of Gothenburg, and Sahlgrenska University Hospital, Gothenburg, Sweden. ${ }^{2}$ Dept of Clinical Sciences Lund, Clinical Physiology and Skåne University Hospital, Lund University, Lund, Sweden. ${ }^{3}$ Cardiology Unit, Dept of Medicine, Karolinska Institutet, Stockholm, Sweden. ${ }^{4}$ Division of Diagnostics and Specialist Medicine, Dept of Health, Medicine and Caring Sciences, and Dept of Clinical Physiology, Linköping University, Linköping, Sweden. ${ }^{5}$ Dept of Medicine Solna, Karolinska Institute, Stockholm, Sweden. ${ }^{6}$ Dept of Respiratory Medicine and Allergy, Karolinska University Hospital, Stockholm, Sweden. ${ }^{7}$ Dept of Public Health and Clinical Medicine, Sundsvall Research Unit, Umeå University, Umeå, Sweden. ${ }^{8}$ Dept of Clinical Sciences Lund, Cardiology, Lund University, Skåne University Hospital, Lund, Sweden. ${ }^{9}$ Dept of Public Health and Clinical Medicine, Heart Centre, Umeå University, Umeå, Sweden. ${ }^{10}$ Dept of Medical Sciences, Cardiology, Uppsala University, and Uppsala Academic Hospital, Uppsala, Sweden. ${ }^{11}$ Dept of Clinical Sciences Lund, Section of Rheumatology, Lund University, Skåne University Hospital, Lund, Sweden.
\end{abstract}

Corresponding author: Clara Hjalmarsson (clara.hjalmarsson@vgregion.se)
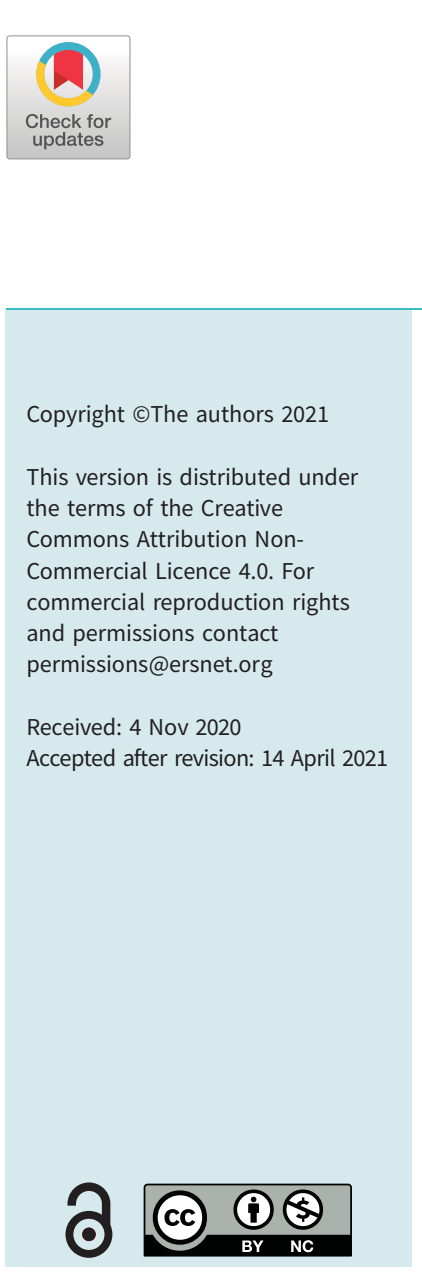

Shareable abstract (@ERSpublications)

ESC/ERS risk assessment accurately identifies low-risk patients but underestimates the 1-year mortality rate of CTD-PAH and IPAH patients assessed as having intermediate risk at diagnosis https://bit.ly/3dDl5x8

Cite this article as: Hjalmarsson C, Kjellström B, Jansson K, et al. Early risk prediction in idiopathic versus connective tissue disease-associated pulmonary arterial hypertension: call for a refined assessment. ERJ Open Res 2021; 7: 00854-2020 [DOI: 10.1183/23120541.00854-2020].

\section{Abstract}

Despite systematic screening and improved treatment strategies, the prognosis remains worse in patients with connective tissue disease-associated pulmonary arterial hypertension (CTD-PAH) compared to patients with idiopathic/hereditary pulmonary arterial hypertension (IPAH). We aimed to investigate differences in clinical characteristics, outcome and performance of the European Society of Cardiology (ESC)/ European Respiratory Society (ERS) risk stratification tool in these patient groups.

This retrospective analysis included incident patients with CTD-PAH ( $\mathrm{n}=197$, of which 64 had interstitial lung disease, ILD) or IPAH ( $\mathrm{n}=305)$ enrolled in the Swedish PAH Register (SPAHR) 2008-2019. Patients were classified as low, intermediate or high risk at baseline, according to the "SPAHR-equation". One-year survival, stratified by type of PAH, was investigated by Cox proportional regression.

At baseline, CTD-PAH patients had lower diffusing capacity for carbon monoxide and lower haemoglobin but, at the same time, lower N-terminal prohormone-brain natriuretic peptide, longer 6 min walk distance, better haemodynamics and more often a low-risk profile. No difference in age, World Health Organisation functional class (WHO-FC) or renal function between groups was found. One-year survival rates were 75, 82 and $83 \%$ in patients with CTD-PAH with ILD, CTD-PAH without ILD and IPAH, respectively. The 1-year mortality rates for low-, intermediate- and high-risk groups in the whole cohort were 0, 18 and 34\% $(\mathrm{p}<0.001)$, respectively. Corresponding percentages for CTD-PAH with ILD, CTD-PAH without ILD and IPAH patients were: $0,26,67 \%$ ( $\mathrm{p}=0.008) ; 0,19,39 \%$ ( $\mathrm{p}=0.004)$; and $0,16,29 \%$ ( $\mathrm{p}=0.001)$, respectively.

The ESC/ERS risk assessment tool accurately identified low-risk patients but underestimated the 1-year mortality rate of CTD-PAH and IPAH patients assessed as having intermediate risk at diagnosis.

\section{Introduction}

Pulmonary arterial hypertension (PAH) is a disease affecting the pulmonary circulation by vascular remodelling resulting from proliferation and migration of pulmonary arterial smooth muscle cells into 
peripheral non-muscular arteries, thickening of the intimal and/or medial layer of muscular arteries, and vaso-occlusive lesions [1]. Based on aetiology, PAH is categorised as idiopathic or hereditary PAH (IPAH) and associated PAH (APAH), where PAH associated with connective tissue disease (CTD-PAH) is the second most prevalent type of PAH [2].

PAH is a serious complication in CTD and is associated with a highly unfavourable prognosis [3-6]. Despite improvement of functional parameters and survival rate by modern treatment $[7,8]$, the outcome of patients with CTD-PAH, and especially of those with systemic sclerosis associated PAH (SSc-PAH), remains poor [9-11].

SSc, the most common underlying aetiology in patients with CTD-PAH, is associated with complex vascular, pulmonary and cardiac pathogenic effects; it may cause pre-capillary pulmonary hypertension (mediated through vascular and interstitial lung changes) [12], rarefication of pulmonary capillaries and/or veno-occlusive disease [13], as well as post-capillary pulmonary hypertension (due to systolic or diastolic dysfunction) [14, 15], resulting in distinct or combined phenotypes, with various prognoses.

The ESC and the ERS advocate a goal-oriented treatment approach for patients with PAH [16, 17]. It is based on serial comprehensive risk assessments that aim to discriminate patients with low, intermediate or high risk of mortality [18-20].

Even though the clinical utility of the risk assessment tool in patients with SSc-PAH has been suggested by two previous publications [21, 22], it has not yet been validated in CTD-PAH, and comparative data on its prognostic value for survival in patients with/without interstitial lung disease (ILD) are lacking.

In a recent post hoc analysis [8] of patients with CTD-PAH included in the AMBITION study, two models of simplified risk assessment at baseline were shown to predict subsequent clinical failure events, but their predictive utility at follow-up (week 16) was limited.

The aim of the present study was to compare clinical characteristics, outcome and 1-year mortality prediction by the ESC/ERS risk stratification tool, according to the "SPAHR-equation" [20], in patients with incident CTD-PAH, with or without ILD, to those with IPAH.

\section{Methods}

\section{Study population}

Adult, incident patients diagnosed with CTD-PAH or IPAH between January 2008 and March 2019 and recorded in the Swedish PAH Register (SPAHR) were included in the study. The PAH diagnosis was confirmed by right heart catheterisation according to the European Society of Cardiology and European Respiratory Society (ESC/ERS) guidelines for the diagnosis and treatment of pulmonary hypertension effective at the time of diagnosis [16, 17, 23]. The date when the patients underwent the right heart catheterisation was defined as baseline. Expert rheumatologists confirmed the CTD diagnosis.

The CTD-PAH group was categorised according to presence or absence of ILD. ILD is reported in SPAHR based on the diagnosis made in the clinics by routine investigations that include pulmonary function tests (collecting data on total lung capacity (TLC), forced vital capacity (FVC), diffusing capacity of the lung for carbon monoxide $\left(D_{\mathrm{LCO}}\right)$, forced expiratory volume in $1 \mathrm{~s}\left(\mathrm{FEV}_{1}\right)$ ) and baseline high-resolution computed tomography of the chest.

Creatinine levels were used to estimate glomerular filtration rate (eGFR) according to the Modification of Diet in Renal Disease (MDRD) formula [24]. Body mass index (BMI) was calculated as weight (expressed in kilograms)/height ${ }^{2}$ (expressed in metres).

\section{The Swedish PAH Register}

SPAHR was launched in 2008, and it constitutes an open continuous register to which all Swedish PAH centres report their data, thereby enabling a high national coverage of $>90 \%$ [25]. The register includes information on demographics, height and weight, comorbidities (hypertension, diabetes mellitus, atrial fibrillation, previous stroke, ischaemic heart disease and thyroid disease, at time of diagnosis), PAH-specific treatments, World Health Organisation functional class (WHO-FC), 6-min walk distance (6MWD), blood biochemistry, and data from echocardiography and right heart catheterisation. SPAHR is approved by the National Board of Health and Welfare and the Swedish Data Protection Authority. All patients were informed about their participation in SPAHR and had the right to decline. The present study complies with the Declaration of Helsinki and was approved by the national ethics committee in Sweden (Dnr. 1002/15 and Dnr. 2019-01065). 


\section{Risk assessment}

Risk assessment was based on specific variables, according to the risk assessment instrument from the ESC/ ERS 2015 guidelines [17] and calculated by using the "SPAHR-equation" [20], which includes data on: WHO-FC, 6MWD, N-terminal prohormone-brain natriuretic peptide (NTproBNP), right atrial area, mean right atrial pressure, pericardial effusion, cardiac index and mixed venous oxygen saturation $\left(S_{\mathrm{vO} 2}\right)$. Each variable was graded from 1 to 3 where 1="low risk", 2=“intermediate risk" and 3="high risk", and the sum of all grades was divided by the number of available variables for each patient rendering a mean grade. The mean grade was rounded off to the nearest integer, which was used to define the patient's risk group. Follow-ups performed after 6-18 months from baseline were included as 1-year follow-up. If multiple follow-ups were registered within the given time frame, the follow-up closest to 12 months was chosen.

\section{Statistics}

Baseline characteristics are reported as percentages for categorical variables and as mean $\pm \mathrm{SD}$ or median (IQR), as appropriate, for continuous variables. $\chi^{2}$-test was used to compare categorical variables; for continuous data, between-group differences were compared using Wilcoxon-Mann-Whitney $U$ test. Risk group, age, sex, PAH type, BMI, eGFR and comorbidities were tested in univariate analyses, and if the p-value was $<0.15$, they were used as covariates in the Cox proportional regression.

Cox proportional hazard analysis was used to calculate hazard ratios for 1-year mortality, and only complete cases were included.

Survival was investigated using the Kaplan-Meier method and the log-rank test. Analyses were stratified by PAH type and/or risk group. Potential interaction effects between PAH type and risk group were assessed. Since there were no events among the low-risk patients during follow-up, this group could not be used as reference in the Cox proportional hazard analysis. Patients were censored at time of lung transplantation, upon death or on January 31, 2019.

Results are presented as the hazard ratio (HR) with 95\% confidence intervals. Change in risk group from baseline to follow-up in relation to PAH type was compared by the Wilcoxon signed-rank test.

p-values $<0.05$ were regarded as statistically significant (2-sided test). All statistical analyses were performed using SPSS Statistical Software Package, version 25.0 (SPSS Inc., Chicago, IL, USA).

Results

The study included 502 patients with PAH (IPAH=305, SSc-PAH=161, other CTD-PAH=36). ILD was reported in 64 CTD-PAH patients, of which 59 had SSc-PAH. Among patients with IPAH, 31 (10.2\%) fulfilled the criteria as responders to an acute vaso-reactivity test [16, 17] and were treated with a high-dose calcium channel blocker. PAH-specific treatment was initiated in $87 \%$ of patients with IPAH and in $89 \%$ of patients with CTD-PAH at baseline. For the remaining patients, first treatment recorded in SPAHR occurred later than 3 months after diagnosis. The median time from baseline to 1-year follow-up was 10 months (IQR 6 months), with no difference between the IPAH and CTD-PAH groups.

\section{Baseline characteristics by PAH type}

The median age was 68 years in both groups, but women were more frequent among the CTD-PAH patients $(78 \%$ versus 57\%, $\mathrm{p}<0.001)$. At baseline, patients with CTD-PAH had longer 6MWD, lower NTproBNP, lower mean pulmonary arterial pressure and pulmonary vascular resistance, and higher cardiac index compared to patients with IPAH (table 1). Lower $D_{\text {LCO }}$ was found among patients with CTD-PAH, whereas patients with IPAH more often had hypertension, diabetes and higher BMI. Haemoglobin was significantly lower among patients with CTD-PAH than IPAH (table 1) and significantly lower in women than in men, in both groups (IPAH: $141 \mathrm{~g} \cdot \mathrm{L}^{-1}$ versus $151 \mathrm{~g} \cdot \mathrm{L}^{-1}, \mathrm{p}<0.001$; CTD-PAH: $131 \mathrm{~g} \cdot \mathrm{L}^{-1}$ versus $138 \mathrm{~g} \cdot \mathrm{L}^{-1}, \mathrm{p}=0.008$ ). No differences in WHO-FC, renal function or PAH-targeted therapy were found between the groups. Patients with IPAH were more often treated with diuretics and anticoagulants compared to CTD-PAH. Characteristics for patients with CTD-PAH, with and without ILD, are shown in table 2. Apart from worse results on the pulmonary function tests, and more pronounced desaturation after performing the 6MWT (lower median oxygen saturation $85 \%$ versus $90 \%, \mathrm{p}=0.039$ ), the patients with ILD had similar characteristics to those without ILD.

\section{Risk assessment}

Patients with CTD-PAH more often had a low-risk profile $(\mathrm{p}=0.011)$ at baseline than patients with IPAH (figure 1a); no difference in risk distribution between CTD-PAH patients with and without ILD was found (figure 2a). At 1-year follow-up, $40 \%$ of patients with IPAH had improved from the intermediate- or 
TABLE 1 Baseline clinical, laboratory and haemodynamic characteristics of the study cohort by PAH-type

\begin{tabular}{|c|c|c|c|c|}
\hline & IPAH & CTD-PAH & Total & $\mathrm{p}$-value \\
\hline Subjects $n$ & 305 & 197 & 502 & \\
\hline \multicolumn{5}{|l|}{ Demography and clinical data } \\
\hline Age years & $68(20)$ & $68(11)$ & $68(16)$ & 0.769 \\
\hline Female gender $\%$ & 57 & 78 & 65 & $<0.001$ \\
\hline $\mathrm{BMI} \mathrm{kg} \cdot \mathrm{m}^{-2}$ & $26(6.5)$ & $24(5.8)$ & $25(6)$ & $<0.001$ \\
\hline WHO-FC (I/II/III/IV) & $1 / 15 / 74 / 10$ & $1 / 19 / 67 / 13$ & $1 / 17 / 71 / 11$ & 0.307 \\
\hline 6MWD m & $250(211)$ & $288(207)$ & $267(210)$ & $<0.001$ \\
\hline SBP mmHg & $130(32)$ & $131(31)$ & $130(31)$ & 0.906 \\
\hline DBP mmHg & $75(21)$ & $75(15)$ & $75(19)$ & 0.722 \\
\hline eGFR mL/min/1.73 m² & $64(35)$ & $67(34)$ & $65(35)$ & 0.796 \\
\hline $\mathrm{Hb} g \cdot \mathrm{L}^{-1}$ & $147(24)$ & $132(23)$ & $141(27)$ & $<0.001$ \\
\hline NTproBNP ng. $\mathrm{L}^{-1}$ & $1792(3354)$ & $1245(3081)$ & $1573(3077)$ & 0.004 \\
\hline$D_{\mathrm{LCO}} \%$ pred mean $\pm \mathrm{SD}$ & $49 \pm 22$ & $41 \pm 15$ & $46 \pm 20$ & $<0.001$ \\
\hline \multicolumn{5}{|l|}{ Comorbidity (\%) } \\
\hline Systemic hypertension & 44 & 35 & 40 & 0.035 \\
\hline Diabetes mellitus & 25 & 10 & 19 & $<0.001$ \\
\hline Ischaemic stroke & 5 & 5 & 5 & 0.843 \\
\hline Ischaemic heart disease & 14 & 16 & 15 & 0.712 \\
\hline Atrial fibrillation & 14 & 11 & 13 & 0.435 \\
\hline Interstitial lung disease & 0 & 32 & 13 & $<0.001$ \\
\hline Obesity & 25 & 11 & 19 & $<0.001$ \\
\hline Kidney dysfunction & 51 & 48 & 49 & 0.547 \\
\hline \multicolumn{5}{|l|}{ Haemodynamics } \\
\hline MRAP mmHg & $8(6)$ & $5(7)$ & $7(7)$ & $<0.001$ \\
\hline MPAP mmHg & $48(13)$ & $38(15)$ & $45(16)$ & $<0.001$ \\
\hline PCWP mmHg & $9(6)$ & $8(6)$ & $8(5)$ & 0.012 \\
\hline $\mathrm{Cl} \mathrm{L} \cdot \mathrm{min}^{-1} \cdot \mathrm{m}^{-2}$ & $2.2(0.8)$ & $2.4(1.0)$ & $2.3(0.8)$ & $<0.001$ \\
\hline PVR Wood units & $10(5)$ & $7(6)$ & $9(6)$ & $<0.001$ \\
\hline $\mathrm{S}_{\mathrm{aO} 2} \%$ & $91(8)$ & $93(6)$ & $92(7)$ & 0.001 \\
\hline $\mathrm{S}_{\mathrm{vO2}} \%$ & $60(13)$ & $64(14)$ & $61(11)$ & $<0.001$ \\
\hline Heart rate bpm & $76(19)$ & $79(22)$ & $77(22)$ & 0.042 \\
\hline \multicolumn{5}{|l|}{ PAH-targeted therapy $(\%)^{\#}$} \\
\hline Single & 65 & 69 & 67 & 0.383 \\
\hline Dual & 21 & 19 & 20 & 0.733 \\
\hline Triple & 1 & 1 & 1 & 0.710 \\
\hline No treatment registered & 13 & 11 & 12 & 0.573 \\
\hline \multicolumn{5}{|l|}{ Supportive therapy (\%) } \\
\hline Anticoagulants & 58 & 42 & 51 & 0.003 \\
\hline Diuretics & 67 & 51 & 61 & $<0.001$ \\
\hline Supplemental oxygen & 32 & 25 & 29 & 0.169 \\
\hline \multicolumn{5}{|l|}{ Risk group } \\
\hline Low/medium/high \% & $12 / 68 / 20$ & $19 / 69 / 12$ & $14 / 69 / 17$ & 0.003 \\
\hline \multicolumn{5}{|l|}{ Transplantation-free survival } \\
\hline 1 year, total (female/male), \% & $83(87 / 79)$ & $80(81 / 74)$ & $82(84 / 78)$ & 0.343 \\
\hline
\end{tabular}

Data are presented as median (interquartile range) or \%, unless otherwise indicated. Bold indicates statistical significance at $p<0.05$. PAH: pulmonary arterial hypertension; IPAH: idiopathic/hereditary pulmonary arterial hypertension; CTD-PAH: connective tissue disease-associated pulmonary arterial hypertension; BMI: body mass index; WHO-FC: World Health Organisation functional class; 6MWD: 6-min walk distance; SBP: systolic blood pressure; DBP: diastolic blood pressure; eGFR: estimated glomerular filtration rate; Hb: haemoglobin; NTproBNP: N-terminal pro-brain natriuretic peptide; $D_{\text {Lco: }}$ diffusing capacity of the lung for carbon monoxide; mRAP: mean right atrial pressure; mPAP: mean pulmonary arterial pressure; PCWP: pulmonary capillary wedge pressure; $\mathrm{Cl}$ : cardiac index; PVR: pulmonary vascular resistance; $\mathrm{S}_{\mathrm{aO} 2}$ : arterial oxygen saturation; $\mathrm{S}_{\mathrm{vO} 2}$ : mixed venous oxygen saturation. ${ }^{\#}$ Started within 3 months from diagnosis.

high-risk group to a lower risk group $(Z=-7.508, p<0.001)$, whereas $8 \%$ were stable, maintaining a low risk. No significant improvement was found among patients with CTD-PAH $(Z=-1.576, p=0.115)$, (figure $1 b$ and c). Subgroup analyses by the presence or absence of ILD showed no difference among CTD-PAH patients with respect to risk stratification at follow-up (figure $2 \mathrm{~b}$ and $\mathrm{c}$ ). The median number of available 
TABLE 2 Baseline clinical, laboratory and haemodynamic characteristics of connective tissue

disease-associated pulmonary arterial hypertension (CTD-PAH) patients, by presence of interstitial lung disease (ILD)

\section{With ILD}

64

\section{Subjects $n$ \\ Demography and clinical data}

Age years

Female gender \%

$\mathrm{BMI} \mathrm{kg} \cdot \mathrm{m}^{-2}$

WHO-FC (I/II/III/IV)

6MWD $\mathrm{m}$

SBP $\mathrm{mmHg}$

DBP $\mathrm{mmHg}$

eGFR $\mathrm{mL} / \mathrm{min} / 1.73 \mathrm{~m}^{2}$

$\mathrm{Hb} g \cdot \mathrm{L}^{-1}$

NTproBNP $\mathrm{ng} \cdot \mathrm{L}^{-1}$

$D_{\text {LCO }} \%$ pred $^{\#}$, mean \pm SD

$\mathrm{FEV}_{1} \%$ pred $^{\#}$, mean \pm sD

FVC $\%$ pred $^{\#}$, mean \pm SD

TLC \% pred" ${ }^{\#}$, mean \pm SD

Comorbidity (\%)

Systemic hypertension

Diabetes mellitus

Ischaemic stroke

Ischaemic heart disease

Atrial fibrillation

Obesity

Kidney dysfunction

Haemodynamics

MRAP $\mathrm{mmHg}$

MPAP $\mathrm{mmHg}$

PCWP $\mathrm{mmHg}$

$\mathrm{Cl} \mathrm{L} \cdot \mathrm{min}^{-1} \cdot \mathrm{m}^{-2}$

PVR Wood units

$\mathrm{S}_{\mathrm{aO} 2} \%$

$\mathrm{S}_{\mathrm{vO} 2} \%$

Heart rate bpm

PAH-targeted therapy (\%)

\section{Single}

Dual

Triple

No treatment registered

Supportive therapy (\%)

Anticoagulants

Diuretics

Supplemental oxygen

Risk group

Low/medium/high \%

Transplantation-free survival

1 year, total (female/male) \%

$68(9)$

72

$24(8)$

$0 / 14 / 67 / 19$

296 (194)

$132(27)$

75 (19)

66 (36)

$134(23)$

1210 (2843)

$36 \pm 13$

$66 \pm 27$

$68 \pm 23$

$72 \pm 18$

\section{6}

13

6

13

9

8

46

4 (6)

36 (18)

7 (5)

$2.6(1.1)$

6 (6)

94 (6)

65 (12)

$83(22)$

13

3

14

36
47
27

36
47

27

$19 / 72 / 9$

$75(76 / 72)$
Without ILD

133

70

$19 / 68 / 14$

$82(83 / 76)$

$6(7)$

39 (13)

$8(6)$

$2.4(0.9)$

$7(6)$

$93(6)$

64 (15)

78 (24)

$68 \quad 0.870$

$23 \quad 0.123$

0

9

0.104

0.326

Data are presented as median (interquartile range) or $\%$, unless otherwise indicated. Bold indicates statistical significance at $\mathrm{p}<0.05$.BMI: body mass index; WHO-FC: World Health Organisation functional class; 6MWD: 6-min walk distance; SBP: systolic blood pressure; DBP: diastolic blood pressure; eGFR: estimated glomerular filtration rate; $\mathrm{Hb}$ : haemoglobin; NTproBNP: N-terminal pro-brain natriuretic peptide; $D_{\mathrm{LCO}}$ : diffusing capacity of the lung for carbon monoxide; $\mathrm{FEV}_{1}$ : forced expiratory volume in $1 \mathrm{~s}$; FVC: forced vital capacity; TLC: total lung capacity; mRAP: mean right atrial pressure; mPAP: mean pulmonary arterial pressure; PCWP: pulmonary capillary wedge pressure; Cl: cardiac index; PVR: pulmonary vascular resistance; $S_{\mathrm{aO} 2}$ : arterial oxygen saturation; $\mathrm{S}_{\mathrm{vo} 2}$ : mixed venous oxygen saturation; PAH: pulmonary arterial hypertension. ${ }^{\#} 35 \%$ missing values among pulmonary functional tests. "Started within 3 months from diagnosis. 

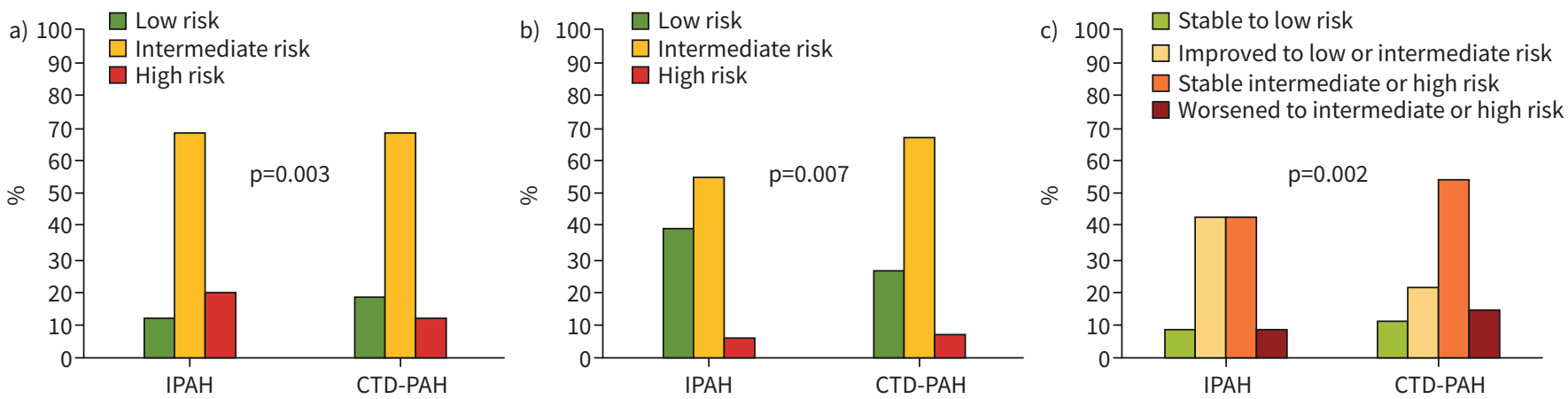

FIGURE 1 Risk assessment presented by type of pulmonary arterial hypertension (PAH). a) Baseline, b) follow-up and c) change in risk group from baseline to follow-up. IPAH: idiopathic and hereditary PAH; CTD-PAH: connective tissue disease-associated PAH.
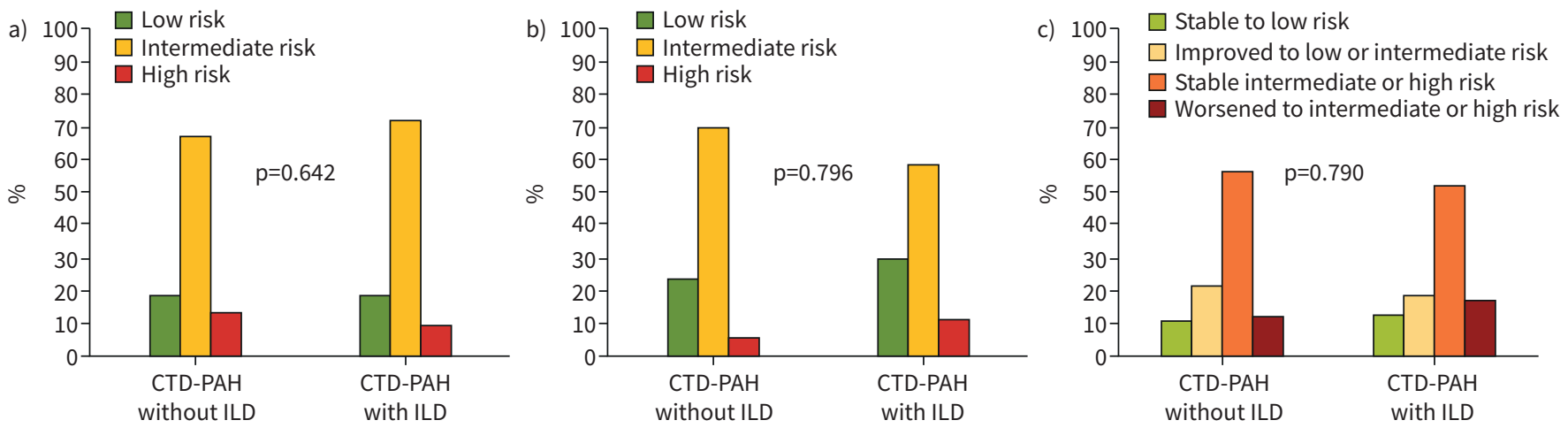

FIGURE 2 Risk assessment presented by presence of interstitial lung disease (ILD) in patients with connective tissue disease-associated pulmonary arterial hypertension (CTD-PAH). a) Baseline, b) follow-up and c) change in risk group from baseline to follow-up.

variables included in the risk assessment was 5 (IQR 5-6) at baseline and 2 (IQR 1-4) at 1-year follow-up. Information regarding PAH-targeted treatment allocation by risk group is shown in table 3.

\section{Transplantation-free survival}

One-year survival rates were 75, 82 and 83\% in patients with CTD-PAH with ILD, CTD-PAH without ILD and IPAH, respectively. In a Cox proportional regression analysis, age (HR 1.04; 95\% CI 1.02-1.07; $\mathrm{p}=0.001$ ), CTD-PAH with ILD (HR 1.98; 95\% CI 1.12-3.53; $\mathrm{p}=0.020$ ) and intermediate- versus high-risk

\section{TABLE 3 Treatment allocation by risk group at baseline and at follow-up (\%)}

\begin{tabular}{|c|c|c|c|}
\hline & \multicolumn{3}{|c|}{ Risk group } \\
\hline & $\begin{array}{c}\text { Low } \\
\text { IPAH/CTD-PAH }\end{array}$ & $\begin{array}{l}\text { Intermediate } \\
\text { IPAH/CTD-PAH }\end{array}$ & $\begin{array}{c}\text { High } \\
\text { IPAH/CTD-PAH }\end{array}$ \\
\hline Baseline & $12 / 19$ & $68 / 69$ & $20 / 12$ \\
\hline Single therapy & $77 / 78$ & $66 / 73$ & $52 / 33$ \\
\hline Combination therapy & $9 / 5$ & $20 / 15$ & $32 / 67$ \\
\hline Follow-up & $43 / 28$ & $52 / 67$ & $5 / 5$ \\
\hline Single therapy & $41 / 56$ & $38 / 38$ & $20 / 14$ \\
\hline Combination therapy & $43 / 33$ & $53 / 57$ & $80 / 86$ \\
\hline
\end{tabular}




\begin{tabular}{|c|c|c|c|}
\hline & $\begin{array}{l}\text { Univariate } \\
\text { p-value }\end{array}$ & $\begin{array}{c}\text { Multivariate } \\
\mathrm{HR}(\mathrm{Cl})\end{array}$ & $p$-value \\
\hline Age & 0.001 & $1.04(1.02-1.07)$ & 0.001 \\
\hline Gender, female & 0.077 & $0.77(0.47-1.27)$ & 0.308 \\
\hline Diabetes mellitus & 0.759 & & \\
\hline Atrial fibrillation & 0.675 & & \\
\hline Ischaemic heart disease & 0.608 & & \\
\hline Ischaemic stroke & 0.546 & & \\
\hline Systemic hypertension & 0.858 & & \\
\hline Risk group at baseline ${ }^{\#}$ & $<0.001$ & $0.42(0.25-0.70)$ & 0.001 \\
\hline Obesity & 0.199 & & \\
\hline Kidney dysfunction & 0.613 & & \\
\hline PAH type & 0.145 & $1.98(1.12-3.53)$ & 0.020 \\
\hline
\end{tabular}

group at baseline (HR 0.42; 95\% CI 0.25-0.70; $\mathrm{p}=0.001$ ) were independent predictors of survival (table 4 and figure 3a). No interaction effect between PAH type and risk group at baseline was found ( $\mathrm{p}=0.177)$.

The mortality rates for low-, intermediate- and high-risk patients in the whole cohort were 0,18 and $34 \%$ $(\mathrm{p}<0.001)$, respectively. Corresponding percentages were 0,26 and $67 \%(\mathrm{p}=0.008)$ for CTD-PAH with ILD; 0, 19 and 39\% ( $p=0.004)$ for CTD-PAH without ILD; and 0, 16 and 29\% ( $p=0.001)$ for IPAH. The results of a separate age- and gender-adjusted Cox proportional regression analysis of patients in intermediate- and high-risk groups are shown in figure $3 \mathrm{~b}$ and c, respectively.

Risk assessment predicted 1-year survival qualitatively, but the mortality rate of patients in the intermediate-risk group was much higher than the one suggested by the ESC/ERS PH-guidelines [16, 17] both for the CTD-PAH and the IPAH patients (figure 4a-c).

\section{Discussion}

In this retrospective longitudinal register study, we investigated the clinical utility of the ESC/ERS risk assessment tool, by use of the "SPAHR-equation", for mortality prediction in incident CTD-PAH patients with or without ILD as compared to IPAH. We found that while the present risk assessment, applied at the time of diagnosis, qualitatively predicted the 1-year survival, it underestimated the mortality rate of both CTD-PAH and IPAH patients assessed as having intermediate risk. Also, despite having better risk profile at baseline, the CTD-PAH patients without ILD had similar 1-year survival to the patients with IPAH, while the survival of CTD-PAH patients with ILD was worse. Finally, clinical improvement, as reflected by reaching a lower risk, was inferior in CTD-PAH patients as compared to IPAH patients during the first year after diagnosis.

The utility of the SPAHR risk stratification model for mortality prediction in patients with CTD-PAH has not been established previously.

In a study including 513 patients with SSc-PAH [22], abbreviated baseline risk assessment based on the number of low-risk criteria, according to the French registry model [18], allowed only modest discrimination of future risk.

In the recently published post hoc analysis of the modified intention to treat CTD-PAH patients included in the AMBITION study [8], an abbreviated version of the SPAHR/COMPERA risk assessment [19, 20] based on three criteria (WHO-FC, NTproBNP and 6MWT), as well as the low-risk noninvasive criteria model used by the French registry [18] were applied. The authors concluded that these abbreviated versions may be useful in predicting PAH-related outcomes in patients with CTD-PAH, but that further research, including large patient cohorts for identifying the most optimal predictive tools, is warranted.

Our results illustrate that risk assessment at baseline underestimates 1-year mortality in both IPAH and CTD-PAH patients with an intermediate risk and does so to a greater extent in the latter group. Conversely, 

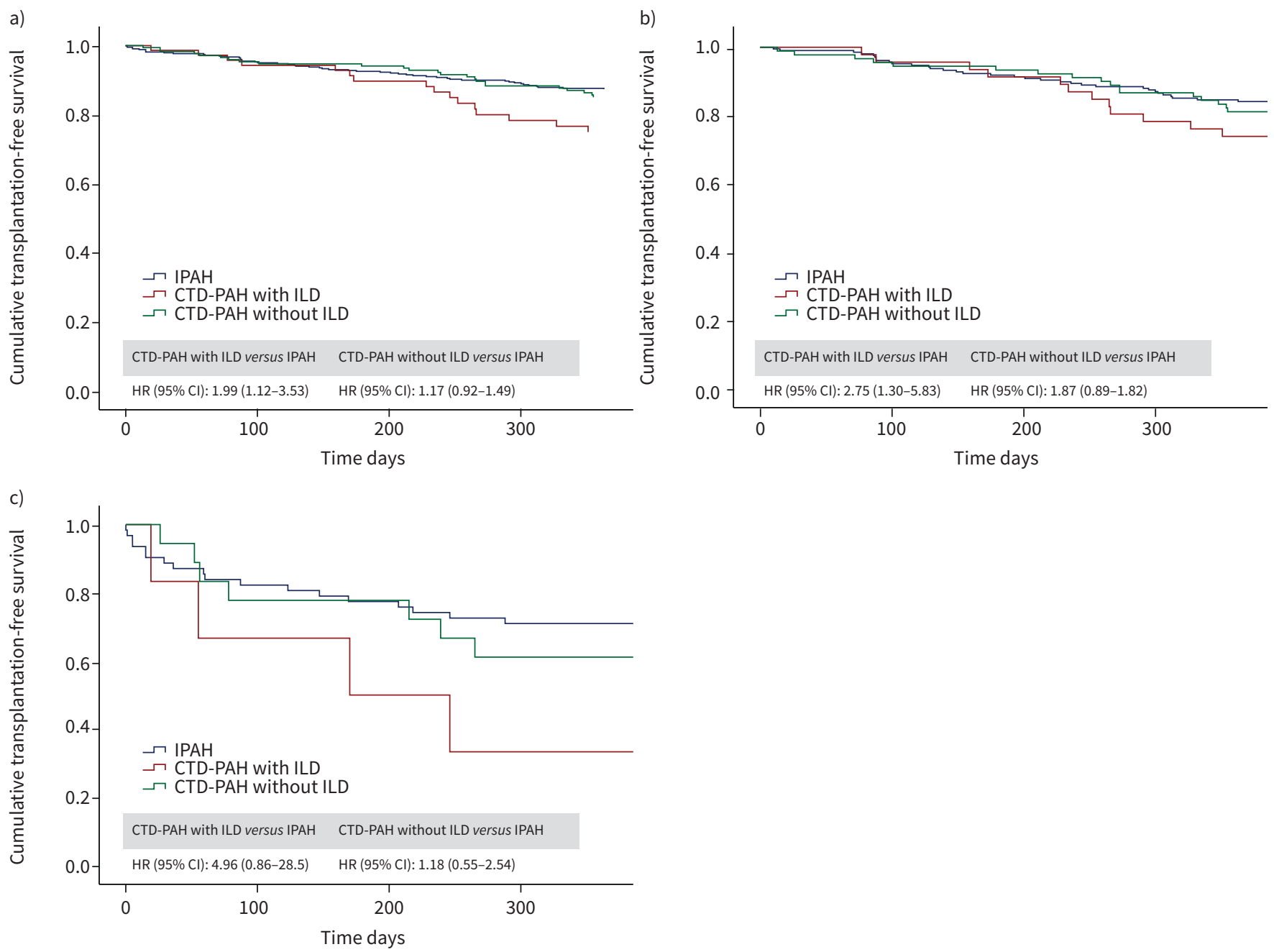

FIGURE 3 Age and gender-adjusted 1-year transplantation-free survival, stratified by type of pulmonary arterial hypertension (PAH). a) The whole study cohort, b) patients in intermediate-risk group and c) patients in high-risk group. IPAH: idiopathic and hereditary PAH; CTD-PAH: connective tissue disease-associated PAH; ILD: interstitial lung disease.

independent of PAH type, no events were reported in patients in the low risk group during the first year after diagnosis. A presumed cause for the IPAH patients with an intermediate risk having a higher mortality rate than the one predicted by the risk assessment tool may be the low percentage of patients receiving up-front PAH combination therapy; an explanation for this might be that a considerable proportion of patients were diagnosed prior to the AMBITION-trial [26], which advocates initial combination treatment. The atypical profile with a higher age and comorbidity burden of patients diagnosed with IPAH and CTD-PAH might be another reason for choosing single over combination therapy [27, 28]. Data regarding up-front combination therapy vary, with some registries reporting higher $[28,29]$ and another lower [30] percentages than SPAHR.

No significant difference in PAH-targeted treatment patterns between the IPAH and the CTD-PAH patients was noted. While the median age at diagnosis was similar in the CTD-PAH and IPAH patients, some differences in demographics, clinical characteristics and outcomes between the groups were obvious; women were more frequent among patients with CTD-PAH, and exercise tolerance as well as haemodynamic data were more favourable in this group; however, $D_{\mathrm{LCO}}$ was lower. At the same time, the comorbidity burden was more pronounced among patients with IPAH. These findings confirm previously published data [5, 11, 31], thereby emphasising the validity of our study cohort.

At baseline, as expected, patients with CTD-PAH and ILD had worse pulmonary function than those without ILD. Apart from this, baseline characteristics were similar between the groups, but survival rate was higher among patients with CTD-PAH without ILD. It is well established that the presence of ILD in 
a)

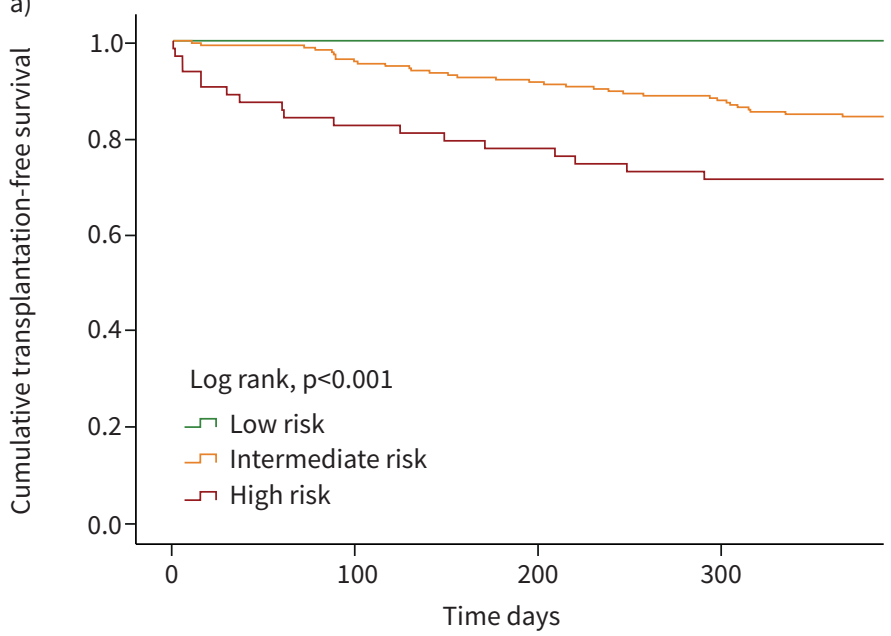

c)

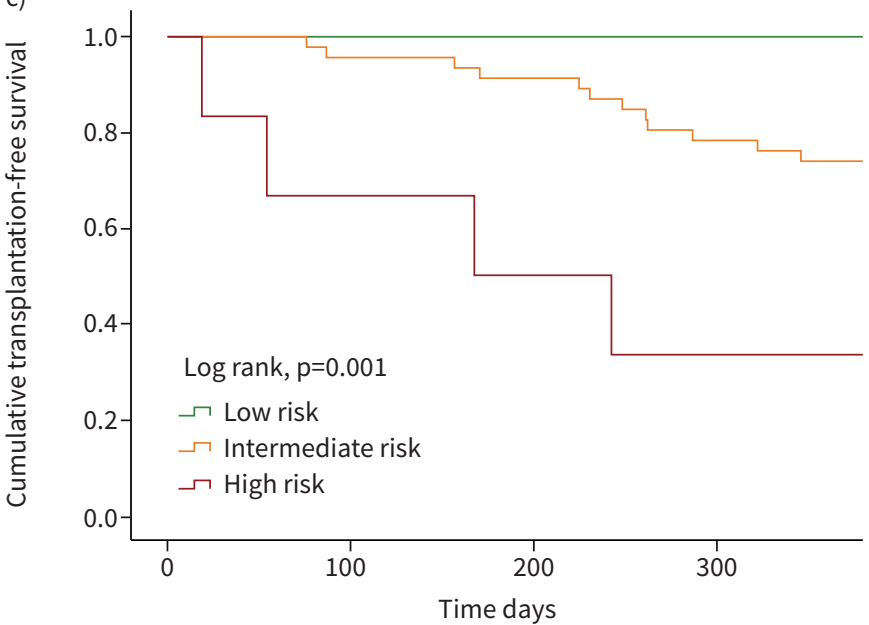

b)

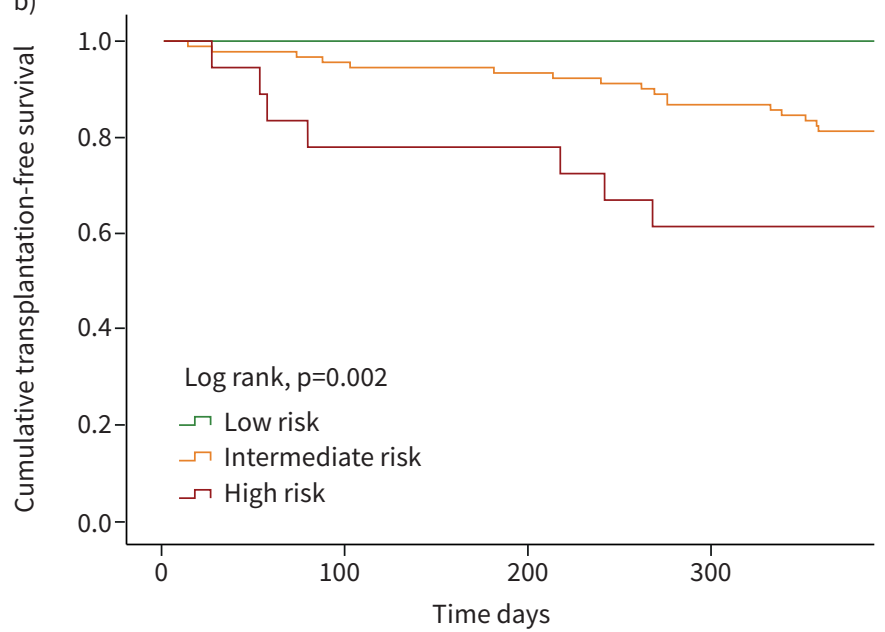

FIGURE 4 Kaplan-Meier curves for time to event by risk category according to the "Swedish PAH Register (SPAHR)-equation" at baseline. a) Idiopathic and hereditary pulmonary arterial hypertension, b) connective tissue disease-associated pulmonary arterial hypertension without interstitial lung disease, c) connective tissue disease-associated pulmonary arterial hypertension with interstitial lung disease.

patients with CTD-PAH makes the prognosis distinctly deleterious [31] with a previously reported pooled 3 -year overall survival rate of $35 \%$ in patients with ILD, as compared to 56\% in those without [32]. At present, due to lack of clear-cut criteria, the discrimination of patients with CTD-PAH and limited ILD from those with moderate ILD and secondary pulmonary hypertension is difficult and mainly based on high-resolution computed tomography and spirometry data [33].

However, including the diagnosis of ILD or the results of pulmonary function tests in the current model for risk assessment of patients with CTD-PAH might be a way to improve the risk estimation.

Notably, 1 year after baseline, only $21 \%$ of the CTD-PAH patients improved, transitioning to low or intermediate risk, while the corresponding percentage was $40 \%$ among the IPAH patients. Thus, treatment goals were not reached in a majority of patients with CTD-PAH. In light of this limited response to therapy, we need to consider how we can improve the treatment and the prognosis for the CTD-PAH population. For many years, the focus has been on systematic screening of SSc patients in order to achieve an early diagnosis of PAH [34]. While this is a commendable effort, additional focus on refined strategies for risk stratification and finding new therapies to improve the outcome of patients with CTD-PAH are needed. Our findings are in line with the recently published results by CHAUvELot et al. [35] showing that response to PAH-specific therapy is poor in patients with SSc-PH and ILD, but not haemodynamically different from that observed in SSc-PAH without ILD. While these findings need to be confirmed in randomised controlled trials, they surely reflect the complexity of CTD, a multifaceted clinical entity leading to a combination of vascular and 
interstitial lung changes, as well as diastolic dysfunction. Moreover, the presence of minor coexisting lung disease on thoracic computed tomography has recently been shown to affect survival in even IPAH patients [36]. Consequently, without an improved phenotyping prior to allocation of treatment, the poor response to targeted PAH-therapy is not unanticipated.

One of the strengths of this study is that it is the first study to investigate the utility of the ESC/ERS risk stratification tool, using the "SPAHR-equation", for mortality prediction in a real-world CTD-PAH cohort. Another strength is that it addresses the impact of ILD on outcome in the context of risk stratification; finally, it includes a relatively large patient population with incident PAH, covering all Swedish PAH centres.

The study is prone to common standard limitations of a register-based descriptive study, such as lack of a standardised study protocol, selection bias with respect to treatment allocation and handling of missing data. For a majority of the patients, not all parameters included in the "SPAHR-equation" were available, but at least functional class, one measure of physical activity level, and one measure of right ventricular function were reported for $>80 \%$ of the study population at baseline and $>50 \%$ at follow-up. Other limitations are related to inherent changes of treatment strategy during the study period, and lack of information on grading of ILD, which is not available in SPAHR.

\section{Conclusion}

In the first year after diagnosis, patients with CTD-PAH, in particular those with ILD, reached treatment goals less often than patients with IPAH. The "SPAHR-equation" underestimated the first-year mortality rate in patients with an intermediate risk, but to a greater extent in patients with CTD-PAH than in those with IPAH.

While the present study highlights the usefulness of a comprehensive risk assessment in CTD-PAH patients, it also endorses the refining of risk stratification strategies, mainly of patients assessed as having intermediate risk, as well as the need of timely escalated therapy, along with that of developing new treatments.

Acknowledgements: We acknowledge the work of the SPAHR registrars at the PAH centres and Uppsala Clinical Research Centre for developing and administering the platform for SPAHR.

Conflict of interest: C. Hjalmarsson reports personal fees from Actelion Pharmaceuticals Sweden AB and Vifor Pharma, grants from MSD, and other support from United Therapeutics, Arena and Acceleron, outside the submitted work. B. Kjellström reports grants from MSD and Actelion Pharmaceuticals Sweden AB outside the submitted work. K. Jansson reports grants, personal fees and other support from Actelion Pharmaceuticals Sweden $A B$ and GlaxoSmithKline, personal fees from Bayer Health Care, and personal fees and other support from MSD, outside the submitted work. M. Nisell reports personal fees from Actelion Pharmaceuticals Sweden $A B$, GlaxoSmithKline, Bayer Health Care, Pfizer and Nordic Infu, and other support from United Therapeutics, outside the submitted work. D. Kylhammar reports grants and personal fees from Actelion Pharmaceuticals Sweden AB, personal fees from GlaxoSmithKline, and grants from Bayer Health Care and Pfizer, outside the submitted work. M. Kavianipour has nothing to disclose. G. Rådegran reports grants, personal fees and other support from Actelion Pharmaceuticals Sweden $A B$ and GlaxoSmithKline, personal fees and other support from Bayer Health Care, personal fees from Nordic Infu, personal fees from Sandoz/Novartis, other from United Therapeutics, other from Pfizer, other from Arena, other from Sanofi-Aventis, other from Acceleron, other from Ely-Lilly, outside the submitted work. S. Söderberg reports personal fees and other support from Actelion Pharmaceuticals Sweden AB outside the submitted work. G. Wikström reports personal fees from Actelion Pharmaceuticals Sweden AB and Orion Pharma, and personal fees and other support from AstraZeneca and Vifor Pharma, outside the submitted work. D.M. Wuttge has nothing to disclose. R. Hesseltrand reports personal fees and other support from Actelion Pharmaceuticals Sweden $A B$ and Boehringer Ingelheim Sweden $A B$, personal fees from Roche Sweden $A B$, and other support from United Therapeutics, outside the submitted work.

Support statement: This study was supported by grants from the Swedish Research Council, the Medical Faculty of Lund University, the Swedish Rheumatism Association, King Gustaf V 80-year Fund, the Österlund Foundation, the Kock Foundation and the EULAR Orphan Disease Programme. The financial supporters had no role in the data collection, analysis or interpretation. Funding information for this article has been deposited with the Crossref Funder Registry.

\section{References}

1 Humbert M, Morrell NW, Archer SL, et al. Cellular and molecular pathobiology of pulmonary arterial hypertension. J Am Coll Cardiol 2004; 43: 12 Suppl. S, 13S-24S. 
2 Humbert M, Sitbon O, Chaouat A, et al. Pulmonary arterial hypertension in France: results from a national registry. Am J Respir Crit Care Med 2006; 173: 1023-1030.

3 Thakkar V, Lau EM. Connective tissue disease-related pulmonary arterial hypertension. Best Pract Res Clin Rheumatol 2016; 30: 22-38.

4 Ramjug S, Hussain N, Hurdman J, et al. Long-term outcomes of domiciliary intravenous iloprost in idiopathic and connective tissue disease-associated pulmonary arterial hypertension. Respirology 2017; 22: 372-377.

5 Kawut SM, Taichman DB, Archer-Chicko CL, et al. Hemodynamics and survival in patients with pulmonary arterial hypertension related to systemic sclerosis. Chest 2003; 123: 344-350.

6 Clements PJ, Tan M, McLaughlin VV, et al. The pulmonary arterial hypertension quality enhancement research initiative: comparison of patients with idiopathic PAH to patients with systemic sclerosis-associated PAH. Ann Rheum Dis 2012; 71: 249-252.

7 Galie N, Corris PA, Frost A, et al. Updated treatment algorithm of pulmonary arterial hypertension. J Am Coll Cardiol 2013; 62: Suppl. 25, D60-D72.

8 Kuwana M, Blair C, Takahashi T, et al. Initial combination therapy of ambrisentan and tadalafil in connective tissue disease-associated pulmonary arterial hypertension (CTD-PAH) in the modified intention-to-treat population of the AMBITION study: post hoc analysis. Ann Rheum Dis 2020; 79: 626-634.

9 Hesselstrand R, Wildt M, Ekmehag B, et al. Survival in patients with pulmonary arterial hypertension associated with systemic sclerosis from a Swedish single centre: prognosis still poor and prediction difficult. Scand J Rheumatol 2011; 40: 127-132.

10 Humbert M, Yaici A, de Groote P, et al. Screening for pulmonary arterial hypertension in patients with systemic sclerosis: clinical characteristics at diagnosis and long-term survival. Arthritis Rheum 2011; 63: 3522-3530.

11 Ramjug S, Hussain N, Hurdman J, et al. Idiopathic and systemic sclerosis-associated pulmonary arterial hypertension: a comparison of demographic, hemodynamic, and MRI characteristics and outcomes. Chest 2017; 152: 92-102.

12 Launay D, Montani D, Hassoun PM, et al. Clinical phenotypes and survival of pre-capillary pulmonary hypertension in systemic sclerosis. PLoS One 2018; 13: e0197112.

13 Dorfmuller P, Montani D, Humbert M. Beyond arterial remodelling: pulmonary venous and cardiac involvement in patients with systemic sclerosis-associated pulmonary arterial hypertension. Eur Respir J 2010; 35: 6-8.

14 de Groote P, Gressin V, Hachulla E, et al. Evaluation of cardiac abnormalities by Doppler echocardiography in a large nationwide multicentric cohort of patients with systemic sclerosis. Ann Rheum Dis 2008; 67: 31-36.

15 Hachulla AL, Launay D, Gaxotte V, et al. Cardiac magnetic resonance imaging in systemic sclerosis: a cross-sectional observational study of 52 patients. Ann Rheum Dis 2009; 68: 1878-1884.

16 Galie N, Humbert M, Vachiery JL, et al. 2015 ESC/ERS Guidelines for the diagnosis and treatment of pulmonary hypertension: The Joint Task Force for the Diagnosis and Treatment of Pulmonary Hypertension of the European Society of Cardiology (ESC) and the European Respiratory Society (ERS): Endorsed by: Association for European Paediatric and Congenital Cardiology (AEPC), International Society for Heart and Lung Transplantation (ISHLT). Eur Respir J 2015; 46: 903-975.

17 Galie N, Humbert M, Vachiery JL, et al. 2015 ESC/ERS Guidelines for the diagnosis and treatment of pulmonary hypertension: The Joint Task Force for the Diagnosis and Treatment of Pulmonary Hypertension of the European Society of Cardiology (ESC) and the European Respiratory Society (ERS): Endorsed by: Association for European Paediatric and Congenital Cardiology (AEPC), International Society for Heart and Lung Transplantation (ISHLT). Eur Heart J 2016; 37: 67-119.

18 Boucly A, Weatherald J, Savale L, et al. Risk assessment, prognosis and guideline implementation in pulmonary arterial hypertension. Eur Respir J 2017; 50: 1700889.

19 Hoeper MM, Kramer T, Pan Z, et al. Mortality in pulmonary arterial hypertension: prediction by the 2015 European pulmonary hypertension guidelines risk stratification model. Eur Respir J 2017; 50: 1700740.

20 Kylhammar D, Kjellstrom B, Hjalmarsson C, et al. A comprehensive risk stratification at early follow-up determines prognosis in pulmonary arterial hypertension. Eur Heart $J$ 2018: 39: 4175-4181.

21 Mercurio V, Diab N, Peloquin G, et al. Risk assessment in scleroderma patients with newly diagnosed pulmonary arterial hypertension: application of the ESC/ERS risk prediction model. Eur Respir J 2018; 52: 1800497.

22 Weatherald J, Boucly A, Launay D, et al. Haemodynamics and serial risk assessment in systemic sclerosis associated pulmonary arterial hypertension. Eur Respir J 2018; 52: 1800678.

23 Galie N, Hoeper MM, Humbert M, et al. Guidelines for the diagnosis and treatment of pulmonary hypertension: the Task Force for the Diagnosis and Treatment of Pulmonary Hypertension of the European Society of Cardiology (ESC) and the European Respiratory Society (ERS), endorsed by the International Society of Heart and Lung Transplantation (ISHLT). Eur Heart J 2009; 30: 2493-2537.

24 Levey AS, Bosch JP, Lewis JB, et al. A more accurate method to estimate glomerular filtration rate from serum creatinine: a new prediction equation. Modification of diet in renal disease study group. Ann Intern Med 1999; 130: 461-470. 

hypertension. N Engl J Med 2015; 373: 834-844.

27 Hjalmarsson C, Radegran G, Kylhammar D, et al. Impact of age and comorbidity on risk stratification in idiopathic pulmonary arterial hypertension. Eur Respir J 2018; 51: 1702310.

28 McGoon MD, Miller DP. REVEAL: a contemporary US pulmonary arterial hypertension registry. Eur Respir Rev 2012; 21: 8-18.

29 Arvanitaki A, Boutsikou M, Anthi A, et al. Epidemiology and initial management of pulmonary arterial hypertension: real-world data from the Hellenic pulmOnary hyPertension rEgistry (HOPE). Pulm Circ 2019; 9: 2045894019877157.

30 Hoeper MM, Pausch C, Grunig E, et al. Idiopathic pulmonary arterial hypertension phenotypes determined by cluster analysis from the COMPERA registry. J Heart Lung Transplant 2020; 39: 1435-1444.

31 Launay D, Humbert M, Berezne A, et al. Clinical characteristics and survival in systemic sclerosis-related pulmonary hypertension associated with interstitial lung disease. Chest 2011; 140: 1016-1024.

32 Lefevre G, Dauchet L, Hachulla E, et al. Survival and prognostic factors in systemic sclerosis-associated pulmonary hypertension: a systematic review and meta-analysis. Arthritis Rheum 2013; 65: 2412-2423.

33 Goh NS, Desai SR, Veeraraghavan S, et al. Interstitial lung disease in systemic sclerosis: a simple staging system. Am J Respir Crit Care Med 2008; 177: 1248-1254.

34 Weatherald J, Montani D, Jevnikar M, et al. Screening for pulmonary arterial hypertension in systemic sclerosis. Eur Respir Rev 2019; 28: 190023.

35 Chauvelot L, Gamondes D, Berthiller J, et al. Hemodynamic response to treatment and outcome in pulmonary hypertension associated with interstitial lung disease versus pulmonary arterial hypertension in systemic sclerosis. Arthritis Rheumatol 2021; 73: 295-304.

36 Peacock AJ, Ling Y, Johnson MK, et al. Idiopathic pulmonary arterial hypertension and co-existing lung disease: is this a new phenotype? Pulm Circ 2020; 10: 2045894020914851. 